\title{
The Dilator-Dotter Technique: A Modified Method of Rapid Internal Carotid Artery Revascularization in Acute Ischemic Stroke
}

\author{
(D) K. Amuluru, (DD. Sahlein, (D) F. Al-Mufti, (D)T. Payner, (D). Kulwin, (D) A. DeNardo, and (D). Scott
}

\begin{abstract}
BACKGROUND AND PURPOSE: Acute ischemic stroke due to tandem occlusive lesions involves high-grade ICA stenosis or occlusion with a distal intracranial occlusion. Several approaches and devices exist in the treatment of tandem occlusions; however, a consensus on the optimal technique does not exist. The Dotter technique is a method of catheter-based angioplasty to recanalize cervical ICA occlusions. We present a modified dilator-Dotter technique, which involves a polyethylene, tapered inner dilator to initially cross the occlusion, followed by guide catheterization and subsequent intracranial thrombectomy. The purpose of this study was to examine the safety and efficacy of this dilator-Dotter technique.
\end{abstract}

MATERIALS AND METHODS: We performed a retrospective review of patients with acute stroke due to tandem ICA intracranial occlusions, treated with our dilator-Dotter technique and thrombectomy between June 2018 and December 2019. We examined clinical, radiographic, and procedural data, as well as complications and outcomes.

RESULTS: Thirty-two patients were included. In 100\% of cases, the dilator-Dotter technique resulted in ICA recanalization allowing successful thrombectomy. $\mathrm{TICl} 2 \mathrm{~b}-3$ revascularization was obtained in 31 patients (96\%), within an average time of 25 minutes. The average preprocedural ICA stenosis was $98 \%$, including 23 patients $(72 \%)$ with complete occlusion. Following the dilator-Dotter technique, average ICA stenosis improved to $59 \%$. There were no instances of death, iatrogenic dissection, emboli to new territory, or symptomatic intracranial hemorrhage.

CONCLUSIONS: The dilator-Dotter technique may be a safe and effective modification of the traditional Dotter technique in allowing rapid ICA recanalization in cases of acute ischemic stroke due to tandem occlusions.

ABBREVIATIONS: ADAPT = direct aspiration first-pass technique; AIS = acute ischemic stroke; ICH = intracranial hemorrhage; LVO = large-vessel occlusion; sICH = symptomatic intracranial hemorrhage

A cute ischemic stroke (AIS) due to tandem occlusive lesions involves acute dissection or ulcerated rupture of an unstable proximal ICA atherosclerotic plaque causing stenosis/occlusion and subsequent embolic intracranial large-vessel occlusion (LVO). ${ }^{1,2}$ Several methods exist in the treatment of AIS due to tandem occlusions, involving various approaches

Received April 13, 2020; accepted after revision June 15.

From the Division of Neurointerventional Radiology (K.A., D.S., A.D., J.S.) and Division of Cerebrovascular Neurosurgery (T.P., C.K.), Goodman Campbell Brain and Spine, Indianapolis, Indiana; Department of Neuroendovascular Surgery and Neurocritical Care (F.A.-M.), Westchester Medical Center at New York Medical College, Valhalla, New York; and Division of Neurointerventional Radiology (K.A., D.S., A.D., J.S.) and Division of Cerebrovascular Neurosurgery (T.P., C.K.), Ascension St. Vincent's Medical Center, Indianapolis, Indiana.

Please address correspondence to Krishna Amuluru, MD, Goodman Campbell Brain and Spine, Division of Neurointerventional Radiology, 13345 Illinois St, Carmel, IN 46032; e-mail: kamuluru@goodmancampbell.com; @AmuluruKrishna

(anterograde versus retrograde) and devices; however, a consensus on the optimal technique does not exist. ${ }^{3,4}$ One of the primary issues in cases of tandem occlusions is management of the proximal ICA occlusion. Balloon angioplasty, emergent carotid stent placement, and catheter angioplasty are all options to recanalize the ICA or achieve access to the distal intracranial occlusion.

The Dotter technique is a well-known method of catheterbased angioplasty used by interventional radiologists in other parts of the body, particularly in peripheral vascular occlusive disease. ${ }^{5}$ Neurointerventionalists have implemented the Dotter technique in cases of AIS involving cervical ICA occlusions, given its simplicity and timeliness. We describe a modification of the Dotter technique using a polyethylene dilator, which is biomechanically less traumatic compared with the traditional Dotter technique. We present a series of 32 cases of AIS due to cervical ICA occlusive disease with tandem, distal, intracranial LVOs treated with the dilator-Dotter technique, followed by intracranial 
thrombectomy. We review the pathophysiology of tandem occlusions, the controversy surrounding treatment techniques, and the biomechanical benefits of dilator angioplasty. This study received institutional (St. Vincent's) review board approval.

\section{MATERIALS AND METHODS}

\section{Patient Selection and Preprocedural Management}

We performed a retrospective review of our Comprehensive Stroke Center data base of patients with AIS treated with endovascular thrombectomy between June 2018 and December 2019. Patients who demonstrated critical stenosis or occlusions of-ICA origin and tandem ipsilateral intracranial occlusions were included. Patients without clinically relevant ICA steno-occlusive disease and those with proximal disease noncontributory to the distal occlusion were excluded. Cases of AIS with proximal ICA occlusion resulting from dissection were excluded. Cases of proximal ICA occlusion treated with the "traditional" Dotter technique or balloon angioplasty were excluded. Cases of AIS due to posterior circulation pathology were excluded.

All patients with AIS presenting or transferred to our center undergo a noncontrast CT scan of the head and are evaluated by a neurologist who determines whether the patient is a candidate for intravenous tPA. Candidacy for endovascular treatment is determined according to the guidelines set forth by the American Heart Association/American Stroke Association for the Management of Patients with AIS and the Society of Neurointerventional Surgery Standards and Guidelines Committee. ${ }^{6,7}$

Clinical and procedural data including age, sex, NIHSS score on presentation, endovascular devices used, procedural time to definitive revascularization (TICI grade $2 \mathrm{~b}-3$ ), preprocedural severity of the stenosis and/or occlusion, procedural complications, postprocedural hemorrhage, NIHSS score at discharge, and mRS score at follow-up were recorded.

\section{Interventional Technique}

All patients in this series were treated under general anesthesia. Access via the right common femoral artery was used in most cases. In 1 case, right radial access was used due to chronic bilateral femoral arterial occlusion. A 6F, 90-cm, straight-tip Neuron MAX 088 guide catheter (Penumbra) over a $5 \mathrm{~F}$ Berenstein catheter with a 0.035-inch Glidewire (Terumo) was used to select the common carotid artery proximal to the occlusion, and cervical and intracranial angiography was performed. In certain cases, a priori knowledge of the proximal occlusion was determined from the preprocedural CTA. The diagnostic catheter was removed, and the prepackaged Neuron MAX 088 inner dilator was inserted into the guide catheter along with the Glidewire. The proximal occlusion was then crossed with the Glidewire, and the inner dilator was subsequently tracked over the wire and across the occlusion. The Neuron MAX 088 guide catheter was then advanced over the dilator, through the occluded segment, and into the cervical ICA. The Neuron MAX 088 guide catheter was vigorously aspirated during and after removal of the dilator and wire. In most cases, contrast was gently injected through the dilator before its removal to confirm the position within the true lumen and to further define the intracranial tandem occlusion.
Attention was then turned to the intracranial thrombus, which was treated using either a combination stent retriever (Solitaire Platinum, Medtronic; or Embotrap II, Johnson \& Johnson) through an aspiration catheter (SOFIA 5F or 6F, MicroVention; React 71, Medtronic; or JET 7 reperfusion catheter, Penumbra) technique or the direct aspiration first-pass technique (ADAPT) technique according to the operator's discretion. Intracranial thrombectomy was determined successful if the therapy resulted in TICI $2 \mathrm{~b}-3$ recanalization. Adjunctive intra-arterial tPA or antiplatelet medications were not used.

Once intracranial flow was restored, the Neuron Max 088 catheter was retracted proximal to the carotid occlusion under vigorous aspiration. In 2 cases involving particularly severe multifocal ICA calcific atherosclerotic disease, there was pre-emptive concern for possible artery-to-artery embolization with removal of the guide catheter. In these cases, the Neuron MAX 088 was retracted using an embolic protection device positioned in the ICA. The patency of the cervical ICA was re-evaluated with repeat cervical angiography (approximately 10-15 minutes) until the operator was confident that the dilator-Dotter technique would maintain patency or at least provide short-term stability until a more definitive therapy could be performed. If re-occlusion was encountered, balloon angioplasty or emergent carotid stent placement was considered on the basis of the patient's history, clinical presentation, and the risk of vessel injury and/or intracranial hemorrhage.

\section{Postinterventional Management}

Following endovascular intervention, antiplatelet therapy is typically started on postprocedural day 1 after imaging demonstrating the absence of hemorrhage. Postprocedural carotid patency is monitored by carotid Doppler and/or CTA. Depending on a patient's pre- and postprocedural functional status, clinical history, and final infarction volume, definitive carotid recanalization with endarterectomy or carotid stent placement is performed within a timeframe determined by the neurology and neurovascular services. In cases involving subacute complete re-occlusion in the absence of new or worsening clinical symptoms, definitive recanalization is avoided due to a physiologically compensatory circle of Willis.

\section{RESULTS}

Between June 2018 and December 2019, we treated 372 patients with endovascular thrombectomy for AIS at our institution. Three hundred ten patients were excluded due to absent and/or noncontributory proximal ICA disease. Seventeen patients were excluded due to proximal steno-occlusive disease treated with the "traditional" Dotter technique or balloon angioplasty before the intracranial thrombectomy. Thirteen patients were excluded due to proximal ICA dissections, determined either on the preprocedural CTA or via DSA showing classic "flame-shaped" luminal narrowing in the absence of calcific atherosclerotic disease. Thus, 32 patients were included who demonstrated critical stenosis or occlusions of the ICA origin and tandem occlusions of the ipsilateral carotid terminus or middle cerebral artery.

Of the 32 patients, 25 were men (78\%) and 7 were women (22\%). The mean age and NIHSS score at presentation were 63 years and 17 (range, 6-30), respectively. Eleven patients (34\%) received intravenous tPA before the procedure. (On-line Table). 


\section{Procedural Results}

The average time from groin access to definitive intracranial revascularization (TICI 2b-3) was 25 minutes (range, 7-88 minutes). In 18 cases, distal thrombectomy was performed using a combination of an aspiration catheter along with a stent retriever, while aspiration alone (ADAPT technique) was used in 12 cases. In 2 patients, the distal tandem occlusion resolved after a proximal Dotter-dilator technique. Definitive intracranial revascularization (TICI 2b-3) was obtained in 31 patients (97\%), with 18 patients (56\%) achieving full TICI 3 recanalization.

Preprocedural ICA stenosis ranged from $90 \%$ to $100 \%$ (mean, $98 \%$ ), including 23 patients with complete occlusion (72\%). The dilator-Dotter technique resulted in successful access to the cervical ICA and the ability to perform intracranial thrombectomy in $100 \%$ of cases. Following the dilator-Dotter technique, ICA stenosis decreased to $59 \%$ (range, $4 \%-100 \%$ ). Twenty-four patients $(75 \%)$ demonstrated a residual post-Dotter stenosis of $\leq 70 \%$ (range, $4 \%-$ 70\%; mean, 50\%), which was not hemodynamically flow-limiting.

In 6 patients (19\%), acute restenosis with flow limitation or re-occlusion was identified after intracranial thrombectomy and after removing the Neuron MAX guide catheter from the ICA. These 6 patients necessitated further interventions ( 5 stent placements and 1 balloon angioplasty, all with embolic protection devices). There were no instances of iatrogenic dissection or emboli to new territory. There were no procedural complications.

\section{Clinical Outcomes}

No patients died during the immediate inpatient hospitalization. There were no cases of postprocedural symptomatic intracranial hemorrhage (sICH). Four patients (13\%) developed postprocedural hemorrhagic transformation, all of which were asymptomatic hemorrhagic infarction type 1 , petechial hemorrhage. The mean NIHSS score at discharge was 6 (range, 0-20). Patient 15 presented 7 hours after acute onset of a left MCA syndrome, with an NIHSS score of 20. Despite successful recanalization and lack of hemorrhagic transformation, the patient demonstrated no meaningful clinical improvement and was discharged to home hospice care. Patient 26 required an emergent internal carotid artery stent after intracranial thrombectomy, which re-occluded on postintervention day 2 , causing massive cerebral edema necessitating hemicraniectomy, and the patient died on postinfarct day 47 .

In 6 patients (19\%), subacute re-occlusion was discovered on follow-up carotid duplex sonography and/or CTA during the subsequent hospitalization. None demonstrated worsening or new symptoms; thus, none required further intervention.

In 20 patients (63\%), no further carotid intervention was performed during the follow-up period, either due to residual stenosis $<70 \%$ or subacute re-occlusion. In 7 patients, eventual definitive carotid endarterectomy was performed, within a range of 1 day to 6 months following the ictal stroke. One patient underwent carotid stent placement 2 months after the ictal stroke.

\section{DISCUSSION}

Approximately $10 \%-25 \%$ of patients with intracranial LVO will have a concomitant proximal extracranial occlusion, and conversely, $50 \%$ of patients with proximal extracranial occlusion will have a tandem distal intracranial LVO $^{8}$ AIS in patients with tandem lesions has a poor prognosis, with only $2 \%-12 \%$ of patients achieving favorable clinical outcome after systemic thrombolysis. ${ }^{4}$ Intravenous thrombolysis and other combined approaches have limited success in this subgroup of patients. ${ }^{8,9}$ Accordingly, the Multicenter Randomized Clinical trial of Endovascular treatment for Acute ischemic stroke in the Netherlands (MR CLEAN), Endovascular Treatment for Small Core and Anterior Circulation Proximal Occlusion With Emphasis on Minimizing CT to Recanalization Times (ESCAPE), and Endovascular Revascularization With Solitaire Device Versus Best Medical Therapy in Anterior Circulation Stroke Within 8 Hours (REVASCAT) trials, which included 32.3\%, 17\%, and $18.6 \%$ of patients with acute tandem occlusions, respectively, showed treatment effect in favor of thrombectomy compared with medical management. ${ }^{10-12}$

In the treatment of tandem occlusions, controversy exists regarding the order in which to treat the proximal and distal lesions, and consensus on management guidelines does not exist. ${ }^{2,3,13-15}$ Advantages of the retrograde/reverse approach include prioritizing the critical intracranial thrombectomy step, quicker restoration of cerebral blood flow, and the possibility of reducing final cerebral infarction volume. ${ }^{14}$ On the other hand, advantages of proximal recanalization first include subsequent access to the intracranial LVO with larger guide catheters, collateral restoration, theoretic reduction of further embolization, and, in some cases, spontaneous distal recanalization. ${ }^{2,3,13-16}$ Controversy also exists regarding balloon angioplasty alone versus carotid stent placement for the treatment of the proximal lesion. Both techniques have associated risks, including distal emboli propagation and intracerebral hemorrhage. ${ }^{2,14,17,18}$ Although angioplasty alone may restore anterograde flow with a less-invasive procedure, disadvantages compared with stent stabilization include the possibility of "rebound" restenosis or re-occlusion, which may be as high as $16 \% .{ }^{19}$

Percutaneous transluminal catheter angioplasty (Dotter technique) was first described in 1965 by Dr Charles Dotter, who used a coaxial system of rigid polyethylene catheters to recanalize an occluded popliteal artery, resulting in salvage of the limb. ${ }^{20}$ The Dotter technique has since been implemented in the neurointerventional space, where simplified techniques of ICA recanalization are regarded favorably in the time-sensitive environment of AIS. ${ }^{21}$ Woodward et $\mathrm{al}^{21}$ described crossing and sequentially dilating ICA stenoses with a $6 \mathrm{~F}$ diagnostic catheter, followed by advancement of a 6 F Shuttle Sheath (Cook Medical, Bloomington, IN) across the stenosis. However, factors such as stenosis severity, length, eccentricity, stiffness, and vessel angulation can preclude catheter placement, resulting in technical failure. Forceful advancement of a catheter with a large size mismatch to the guidewire (step-off) across the stenosis may result in intimal dissection, flap formation, embolization, perforation, and spasm.

The Neuron MAX 088 guide catheter comes prepackaged with a 100 - or $110-\mathrm{cm}$ (depending on the length of the Neuron MAX 088 chosen) High-density polyethylene (HDPE) polymer inner dilator shaft, which has an inner diameter (ID) of 0.035 inches. Used mainly for femoral artery access directly through the skin, it extends $11.5 \mathrm{~cm}$ distal to the Neuron MAX 088 catheter tip when fully click-locked through the included cross-cut valve (Fig 1). Smooth, coaxial dilation of an occluded ICA using 
the tapered dilator is not only intuitively less traumatic compared with traditional Dotter angioplasty over a wire or nontapered diagnostic catheter, but it also exerts less shear force on the arterial intimal surface and associated plaque. ${ }^{22,23}$ Furthermore, sequentially tapered dilator angioplasty results in less longitudinal movement of the vessel compared with dilation with a sole catheter over a wire with a large size mismatch, reducing the risk of dissection, embolization, and perforation. ${ }^{22}$ Although the risk of iatrogenic embolization exists any time an unstable stenosis is

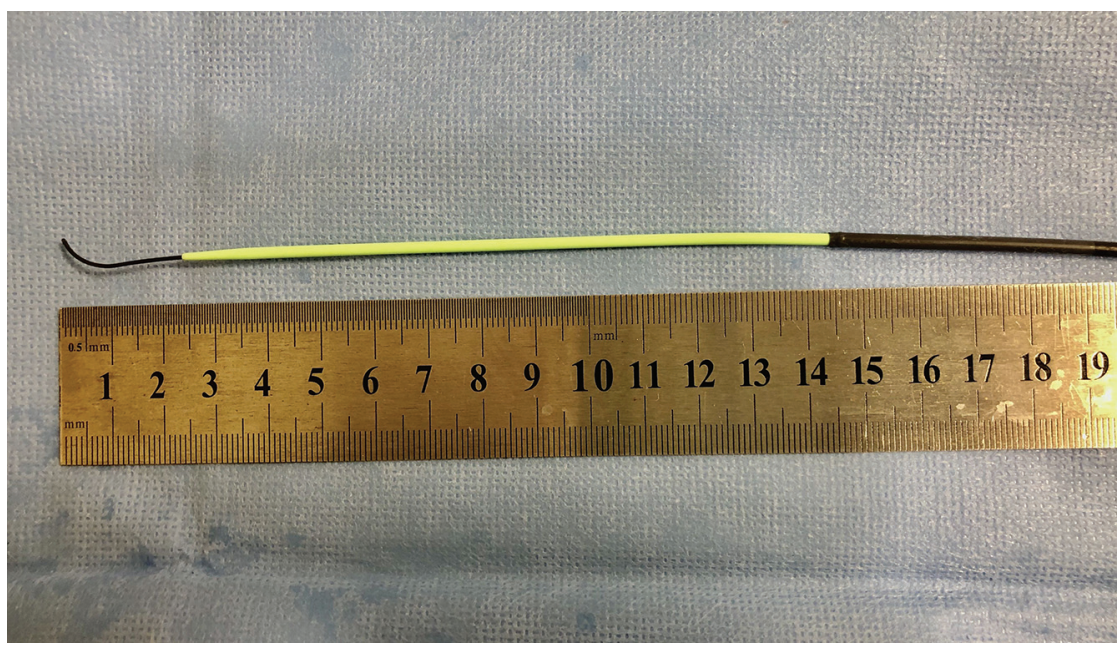

FIG 1. High-density polyethylene polymer inner dilator shaft extends $11.5-\mathrm{cm}$ distal to the Neuron MAX 088 catheter tip, with a 0.035-inch Glidewire. crossed, in our cohort, our technique caused zero cases of dissection, perforation, or embolization.

In our cohort, we encountered zero instances of sICH. This outcome is comparable with previous reports of the traditional Dotter technique in AIS due to tandem occlusions and is favorable compared with data on patients treated with emergent carotid stent placement. ${ }^{3,21,24,25}$ Studies examining the complications of acute ICA stent placement in patients with AIS have shown an intracranial hemorrhage ( $\mathrm{ICH}$ ) rate as high as $25 \%{ }^{3,24,25}$ Dorado et $\mathrm{al}^{25}$ showed that the placement of a stent was an independent predictor of sICH in their report on acute stent placement during stroke intervention. Several theories exist on why emergent carotid stent placement and mechanical thrombectomy may increase the risk of ICH. Patients with increasingly proximal occlusions are at higher risk of established infarction of the basal ganglia, which predisposes to hemorrhagic conversion. Additionally, emergent stent placement exposes patients to hyperperfusion syndrome (re-establishment of flow dynamics that have been chronically absent), which, when combined with established infarction, can lead to an increased risk of ICH. Finally, aggressive prophylactic antiplatelet therapy, to prevent in-stent thrombosis, may further
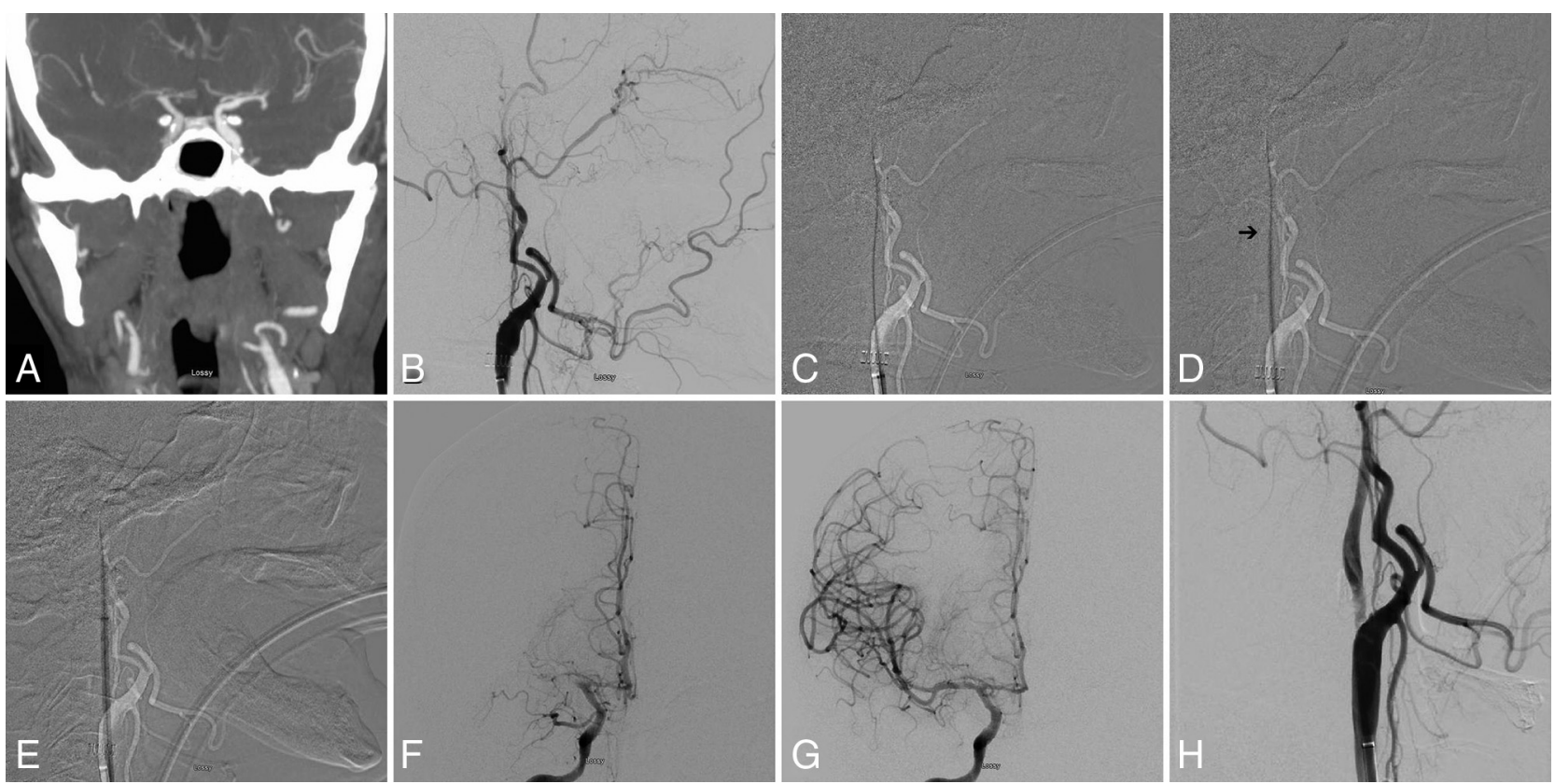

FIG 2. A, Preprocedural CTA showing acute occlusion of right MCA. Note nonopacification of cervical right ICA. B, Digital subtraction angiogram of the right common carotid artery in a lateral view shows occlusion of cervical ICA at the origin. C, Subtracted fluoroscopic roadmap shows crossing of the cervical ICA occlusion with a Glidewire, followed by the Neuron MAX 088 inner dilator $(D)$. Despite radiolucency of the dilator, note the subtle change in Glidewire angulation indicating the dilator tip location (arrow). E, Subsequent dilator-Dotter angioplasty by crossing the ICA occlusion with the Neuron MAX 088. Note the lack of dilator-catheter "step-off." $F$, Angiogram in a frontal view shows tandem intracranial occlusion of right MCA, with subsequent successful thrombectomy $(G)$ yielding a $\mathrm{TICl} 3$ recanalization. $H$, The patient is left with $80 \%$ stenosis on the final postinterventional angiogram, which eventually asymptomatically reoccluded. 

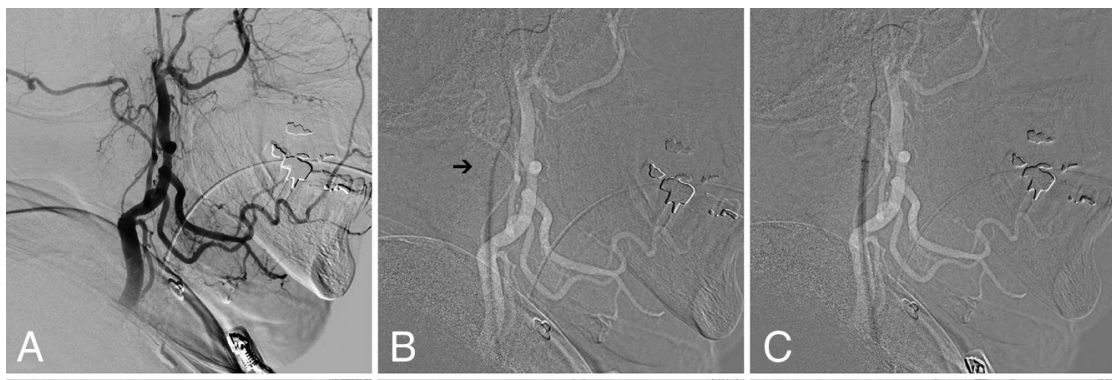

D
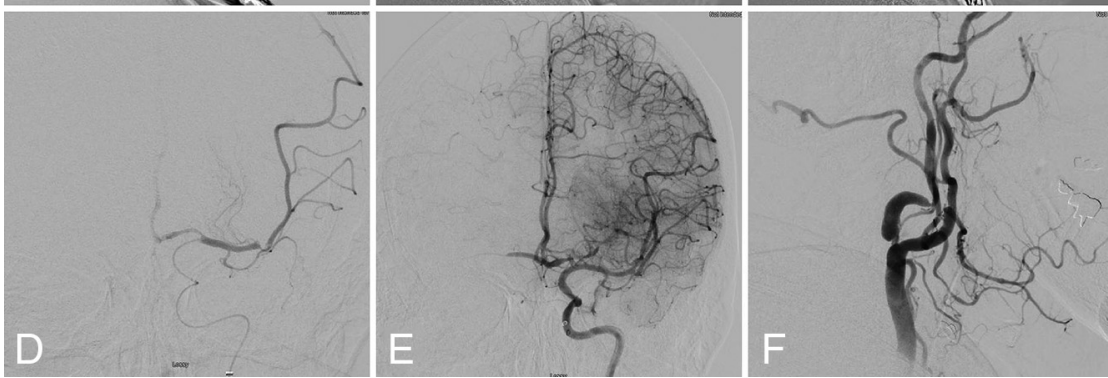

FIG 3. A, Digital subtraction angiogram of the left common carotid artery in a lateral view showing occlusion of cervical ICA at the origin. $B$, Subtracted fluoroscopic roadmap shows crossing of the cervical ICA occlusion with the Glidewire and Neuron MAX 088 inner dilator Note the subtle change in Glidewire angulation indicating the dilator tip location (arrow). C, Subsequent dilatorDotter angioplasty by crossing the ICA occlusion with the Neuron MAX 088. D, Microcatheter angiogram in frontal view shows tandem intracranial occlusion of superior-division M2 segment of the left MCA, with subsequent successful thrombectomy $(E)$ yielding $\mathrm{TICI} 3$ recanalization. Note the robust crossflow across the anterior communicating artery. $F$, The patient was left with high-grade stenosis on the final postinterventional angiogram, which eventually asymptomatically re-occluded on follow-up carotid duplex sonography.

increase subsequent ICH risk. ${ }^{25}$ These issues are only further complicated in patients who have received intravenous $\mathrm{tPA}$ or in whom the cerebral infarct volume is unknown. ${ }^{17}$ Thus, any approach that can restore proximal ICA patency while obviating emergent balloon angioplasty or stent placement is a potentially powerful technique in this population.

In our cohort, we encountered a mean preprocedural stenosis rate of $98 \%$, which improved to $58 \%$ after dilator-Dotter angioplasty. These results compare slightly favorably with those in a recent examination of the traditional Dotter technique, which examined 7 patients with a preinterventional stenosis of $88 \%$, which improved to $61 \%$ after intervention. ${ }^{21}$ In 6 of our patients (19\%), acute rebound stenosis/occlusion necessitated further intervention with balloon angioplasty or stent placement, all of which were successful without complication. Six additional patients (23\%) demonstrated subacute re-occlusion during their hospitalization, all of whom were asymptomatic, presumably due to a physiologically compensatory circle of Willis (Figs 2 and 3). While these 12 patients demonstrate the unclear long-term efficacy of the dilator-Dotter technique, the technique facilitated definitive recanalization in a timely manner (mean, 25 minutes) without the need for ancillary devices and their associated risk. Furthermore, we postulate that in addition to enabling rapid ICA access for timely thrombectomy, the intermediate recanalization achieved may be sufficient to provide short-term stability until more definitive endarterectomy or stent placement can be performed. Indeed,
8 of our patients clinically improved enough to undergo eventual carotid endarterectomy or stent placement.

The predominant risks of the dilator-Dotter technique include iatrogenic dissection, embolization, and/or perforation, though these risks are present when any unstable stenosis is crossed, regardless of the technique or device used. In several cases, once the proximal occlusion was crossed using the dilator-Dotter technique, subsequent placement of the guide catheter in the ICA resulted in flow arrest, decreasing the likelihood of iatrogenic thromboembolization during thrombectomy. Finally, the inner dilator is radiolucent, making visualization difficult. However, if the Neuron MAX 088 is visualized on fluoroscopy, appreciation of the $11.5-\mathrm{cm}$ length extrusion from the Neuron MAX 088 catheter tip allows operator recognition of the tip location. Furthermore, appreciation of the change in angulation of the dilator to the Glidewire will also aid in recognition of tip location (Fig 1). Operators should be aware that all ICA occlusions in this series were in the setting of atherosclerosis; if an occlusion due to dissection is encountered, using the dilator-Dotter technique may exacerbate the dissection.

Limitations of our study include the retrospective nature as well as the small patient population. The results herein reported using the Neuron MAX inner dilator might not be generalizable to other manufacturers' dilators. Given the presence of tandem intracranial LVO, there is no way to determine whether our technique caused angiographically occult embolism due to the pre-existing distal occlusion. Despite this limitation, we encountered zero instances of embolization to new territory when comparing the preprocedural CTA with the first angiogram after the dilator-Dotter technique.

\section{CONCLUSIONS}

In this series of 32 patients with AIS due to tandem ICA intracranial occlusions, the dilator-Dotter technique resulted in rapid ICA recanalization in all cases, allowing successful thrombectomy attempts. There were no instances of death, iatrogenic dissection, emboli to new territory, or symptomatic intracranial hemorrhage. The dilator-Dotter technique may be a safe and effective modification of the traditional Dotter technique in allowing rapid ICA recanalization in cases of AIS due to tandem occlusions.

Disclosures: Daniel Sahlein—UNRELATED: Consultancy: Medtronic, MicroVention, phenox, Stryker; Grants/Grants Pending: MicroVention; Payment for Lectures Including Service on Speakers Bureaus: Medtronic, MicroVention; Payment for Development of 
Educational Presentations: Medtronic. Fawaz Al-Mufti-UNRELATED: Employment. Westchester Medical Center at New York Medical College.

\section{REFERENCES}

1. Kim YS, Garami Z, Mikulik R, et al; CLOTBUST Collaborators. Early recanalization rates and clinical outcomes in patients with tandem internal carotid artery/middle cerebral artery occlusion and isolated middle cerebral artery occlusion. Stroke 2005;36:869-71 CrossRef Medline

2. Spiotta AM, Lena J, Vargas J, et al. Proximal to distal approach in the treatment of tandem occlusions causing an acute stroke. $J$ Neurointerv Surg 2015;7:164-69 CrossRef Medline

3. Malik AM, Vora NA, Lin R, et al. Endovascular treatment of tandem extracranial/intracranial anterior circulation occlusions: preliminary single-center experience. Stroke 2011;42:1653-57 CrossRef Medline

4. Rubiera M, Ribo M, Delgado-Mederos R, et al. Tandem internal carotid artery/middle cerebral artery occlusion: an independent predictor of poor outcome after systemic thrombolysis. Stroke 2006;37:2301-05 CrossRef Medline

5. Brillu C, Picquet J, Villapadierna F, et al. Percutaneous transluminal angioplasty for management of critical ischemia in arteries below the knee. Ann Vasc Surg 2001;15:175-81 CrossRef Medline

6. Powers WJ, Rabinstein AA, Ackerson T, et al. Guidelines for the early management of patients with acute ischemic stroke: 2019 update to the $\mathbf{2 0 1 8}$ guidelines for the early management of acute ischemic stroke: a guideline for healthcare professionals from the American Heart Association/American Stroke Association. Stroke 2019;50:e344-418 CrossRef Medline

7. Mokin M, Ansari SA, McTaggart RA, et al; Society of NeuroInterventional Surgery, Indications for thrombectomy in acute ischemic stroke from emergent large vessel occlusion (ELVO): report of the SNIS Standards and Guidelines Committee. J Neurointerv Surg 2019;11:215-20 CrossRef Medline

8. Christou I, Felberg RA, Demchuk AM, et al. Intravenous tissue plasminogen activator and flow improvement in acute ischemic stroke patients with internal carotid artery occlusion. J Neuroimaging 2002;12:119-23 CrossRef

9. Lekoubou A, Cho TH, Nighoghossian N, et al. Combined intravenous recombinant-tissular plasminogen activator and endovascular treatment of spontaneous occlusive internal carotid dissection with tandem intracranial artery occlusion. Eur Neurol 2010;63:21114 CrossRef Medline

10. Berkhemer OA, Fransen PS, Beumer D, et al; MR CLEAN Investigators. A randomized trial of intraarterial treatment for acute ischemic stroke. N Engl J Med 2015;372:11-20 CrossRef Medline

11. Campbell BC, Mitchell PJ, Kleinig TJ, et al. Endovascular therapy for ischemic stroke with perfusion-imaging selection. $N$ Engl J Med 2015;372:1009-18 CrossRef
12. Jovin TG, Chamorro A, Cobo E, et al; REVASCAT Trial Investigators. Thrombectomy within 8 hours after symptom onset in ischemic stroke. N Engl J Med 2015;372:2296-2306 CrossRef Medline

13. Mpotsaris A, Bussmeyer M, Buchner $H$, et al. Clinical outcome of neurointerventional emergency treatment of extra- or intracranial tandem occlusions in acute major stroke: antegrade approach with Wallstent and Solitaire stent retriever. Clin Neuroradiol 2013;23:20715 CrossRef Medline

14. Rangel-Castilla L, Rajah GB, Shakir HJ, et al. Management of acute ischemic stroke due to tandem occlusion: should endovascular recanalization of the extracranial or intracranial occlusive lesion be done first? Neurosurg Focus 2017;42:E16 CrossRef Medline

15. Al-Mufti F, Amuluru K, Manning NW, et al. Emergent carotid stenting and intra-arterial abciximab in acute ischemic stroke due to tandem occlusion. Br J Neurosurg 2017;31:573-77 CrossRef Medline

16. Amuluru K, Al-Mufti F, Romero CE. Acute ischemic stroke due to common carotid ostial disease with tandem intracranial occlusions treated with thrombectomy and staged retrograde stenting. Interv Neurol 2018;7:445-51 CrossRef Medline

17. Behme D, Molina CA, Selim $\mathrm{MH}$, et al. Emergent carotid stenting after thrombectomy in patients with tandem lesions. Stroke 2017;48:1126-28 CrossRef Medline

18. Stampfl S, Ringleb PA, Mohlenbruch M, et al. Emergency cervical internal carotid artery stenting in combination with intracranial thrombectomy in acute stroke. AJNR Am J Neuroradiol 2014;35:74146 CrossRef Medline

19. McCabe DJ, Pereira AC, Clifton A, et al; CAVATAS Investigators Restenosis after carotid angioplasty, stenting, or endarterectomy in the Carotid and Vertebral Artery Transluminal Angioplasty Study (CAVATAS). Stroke 2005;36:281-86 CrossRef Medline

20. Dotter CT, Judkins MP. Percutaneous transluminal treatment of arteriosclerotic obstruction. Radiology 1965;84:631-43 CrossRef Medline

21. Woodward K, Wegryn S, Staruk C, et al. The Dotter method revis ited: early experience with a novel method of rapid internal carotid artery revascularization in the setting of acute ischemic stroke. $J$ Neurointerv Surg 2016;8:360-66 CrossRef Medline

22. Kinney TB, Chin AK, Rurik GW, et al. Transluminal angioplasty: a mechanical-pathophysiological correlation of its physical mechanisms. Radiology 1984;153:85-89 CrossRef Medline

23. Kinney TB, Fan M, Chin AK, et al. Shear force in angioplasty: its relation to catheter design and function. AJR Am J Roentgenol 1985;144:115-22 CrossRef Medline

24. Cohen JE, Gomori JM, Rajz G, et al. Extracranial carotid artery stenting followed by intracranial stent-based thrombectomy for acute tandem occlusive disease. J Neurointerv Surg 2015;7:412-17 CrossRef Medline

25. Dorado L, Castano C, Millan M, et al. Hemorrhagic risk of emergent endovascular treatment plus stenting in patients with acute ischemic stroke. J Stroke Cerebrovasc Dis 2013;22:1326-31 CrossRef Medline 